\title{
Espundia: a tropical infectious disease making its way to Europe. A case report of nasal mucocutaneous leishmaniasis in the Netherlands*
}

\author{
A.A. Schenck', A.C. Westgeest'ㄹ, E.J.C. Teunissen', E.F. Schippers ${ }^{2,3}$, \\ J.P. Koopman'
}

Rhinology Online, Vol 3: 73 - 78, 2020 http://doi.org/10.4193/RHINOL/20.031

\author{
*Received for publication \\ April 19, 2020 \\ Accepted: May 27, 2020 \\ Published: June 2, 2020
}

\begin{abstract}
Background: We present a case history of a patient with nasal mucocutaneous leishmaniasis, also known as Espundia. Knowledge of this tropical infectious disease is usually limited among otorhinolaryngologists, but is of importance because patients may present with symptoms in nose or mouth.

Case presentation: A 25-year old healthy man was referred to our outpatient clinic with nasal obstruction and swelling at the left part of the nose since two weeks. The patient had travelled to Peru several months ago. After extensive diagnostic testing, mucocutaneous leishmaniasis was diagnosed. Leishmaniasis is a tropical infectious disease caused by a parasite of the Leishmania-family. Depending on the species, it can cause cutaneous, mucocutaneous or visceral disease. Mucocutaneous leishmaniasis, called'Espundia', has a preference for the nasal mucosa and can lead to severe tissue destruction in the nasopharyngeal region. This disease should always be treated.

Conclusion: This case is presented for two reasons. Firstly, knowledge of this disease is important in reducing doctors' delay, because mucocutaneous leishmaniasis may lead to secondary infection and facial deformation. Secondly, due to globalisation and climate change an increase in incidence of leishmaniasis in Europe is expected. Therefore, otorhinolaryngologists will more frequently be confronted with leishmaniasis and should be able to recognise it.
\end{abstract}

Key words: Leishmaniasis, mucocutaneous, nasal obstruction, nasal swelling, parasitology, tropical infectious disease

\section{Introduction}

Nasal obstruction is a common complaint in ear, nose, and throat (ENT-) practice. Frequent causes are infection, anatomical abnormalities (such as septal deviation), mucosal hypersensitivity (allergy) or tumours (benign polyps or a malignancy). However, there are also rarer causes. We present a case of nasal obstruction and outer swelling of the nose caused by leishmaniasis, a tropical infectious disease caused by a parasite of the
Leishmania-family. Leishmaniasis is endemic in areas such as Latin America and the Middle East. However, European otorhinolaryngologists are rarely confronted with this disease. In this case, failure to recognise mucocutaneous leishmaniasis as a possible cause of nasal obstruction led to a treatment delay of four weeks. This case study is aimed at bringing leishmaniasis to your attention, and highlighting the importance of taking a travel history, especially in the case of unexplained symptoms.

ENT: ear-, nose-, throat; CRP: C-reactive protein; ESR: erythrocyte sedimentation rate; AFB: acid-fast bacilli; CT: computed tomography; MRI: magnetic resonance imaging; PET-CT: positron emission tomography-computed tomography; FDG: fludeoxyglucose; PCR: polymerase chain reaction; ML: mucocutaneous leishmaniasis; WHO: World Health Organization 

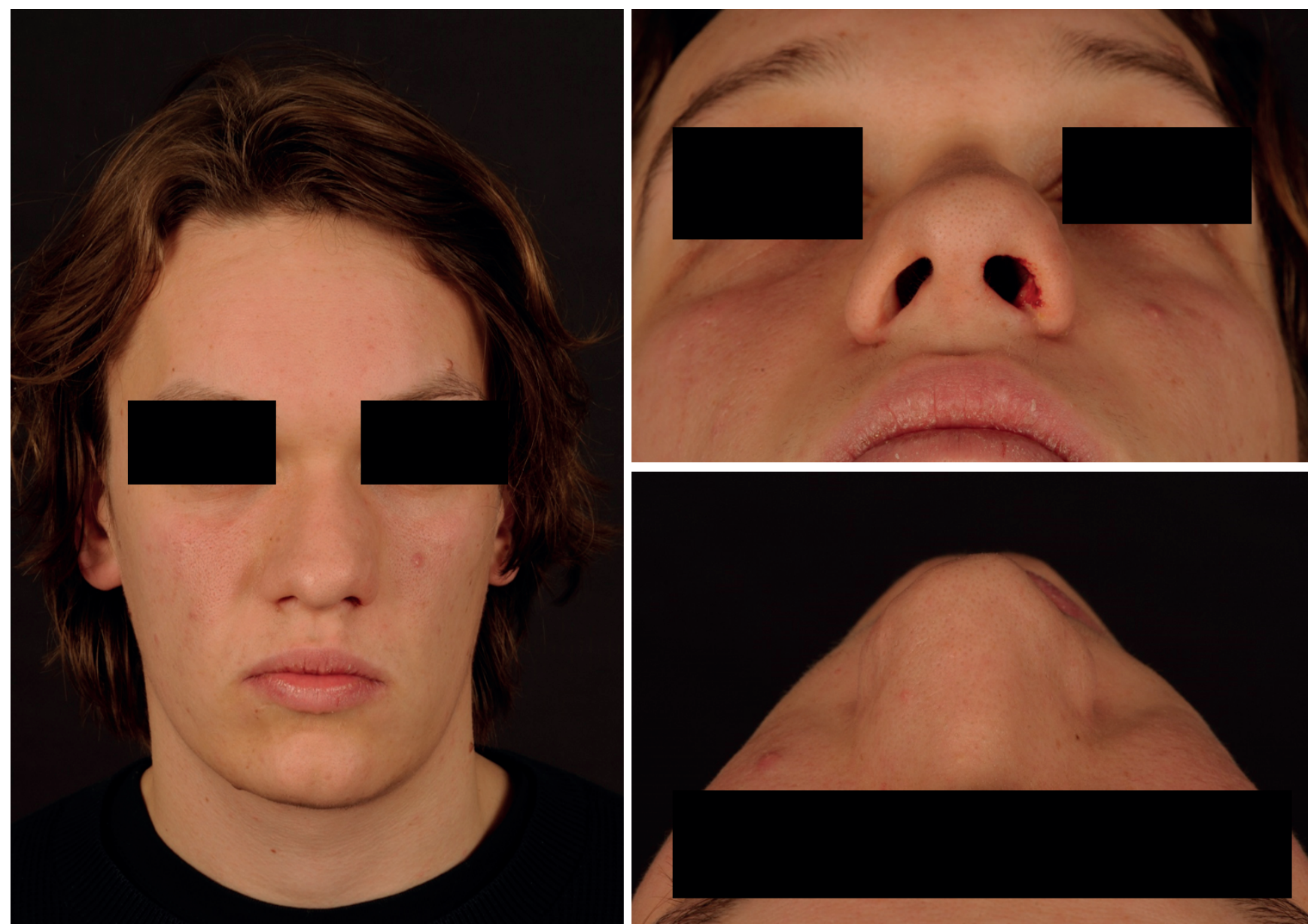

Figure 1. Clinical images taken at the time of presentation. A swelling of the left lateral side of the nose is visible.
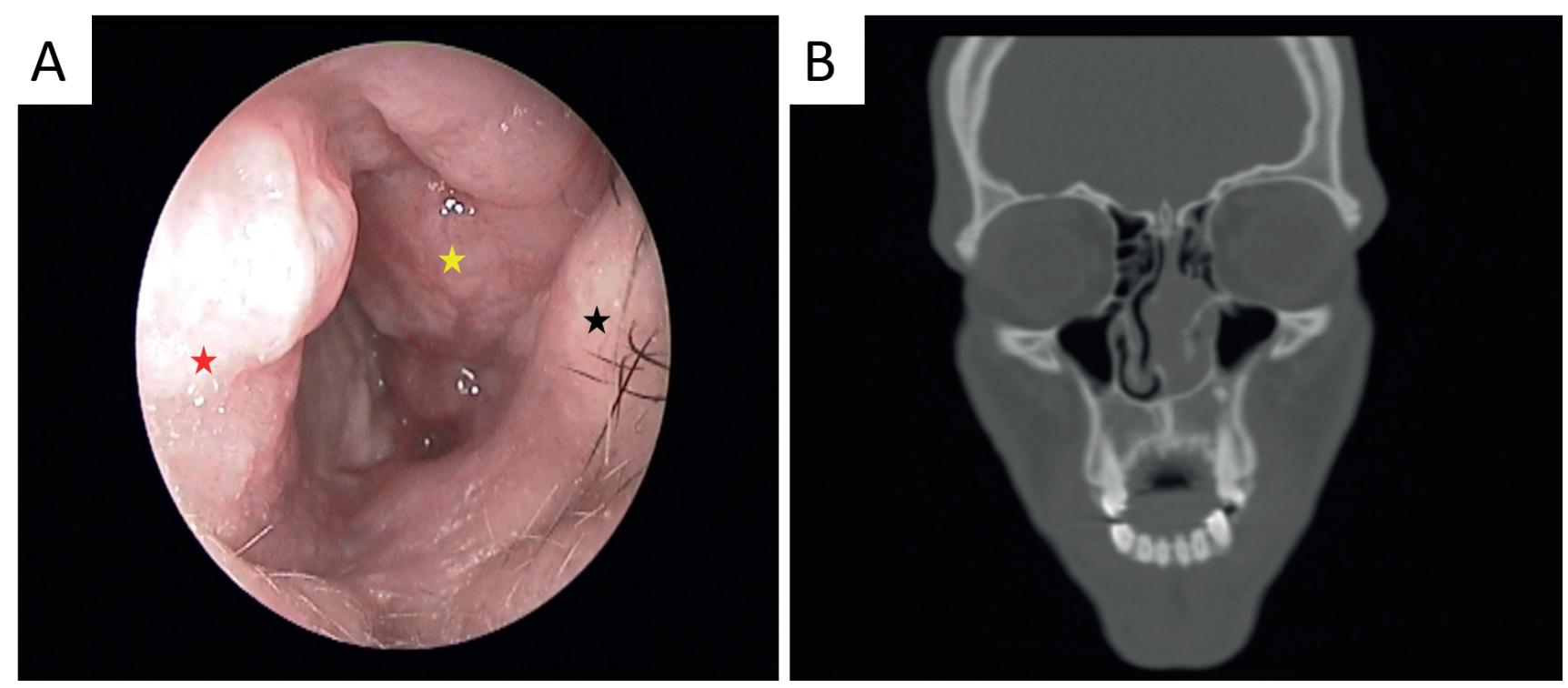

Figure 2. Intranasal image and CT-scan at first consultation. A. Intranasal image of the left nasal cavity. The red asterisk indicates the nasal septum; the yellow asterisk indicate the mucosal tumour that was found superiorly from the inferior turbinate in the nasal cavity (black asterisk). B. CT-scan of the paranasal sinuses: coronal view. Thickening of the soft tissue in the left nasal cavity, with extension to the lateral side. There is no destruction of surrounding tissues. 


\section{Case presentation}

A 25-year-old man, with no prior medical history, was referred to our ENT-outpatient clinic with a painful swelling of the left lateral side of the nose since two weeks. The swelling caused obstruction of the left nasal cavity. There was no history of trauma to the nose, rhinorrhoea or fever. Treatment with antibiotics (amoxicillin $500 \mathrm{mg}$ per day) had already been initiated by his general practitioner, leading to a reduction in pain but not in swelling. At a follow-up visit, the patient mentioned he had travelled to Peru several months earlier, where he had suffered from numerous sandfly bites. On physical examination, an erythematous, firm swelling of the left lateral side of the nose was noted, without fluctuation or oedema. The skin was intact (Figure 1). Intranasally, a red mucosal tumour, superiorly from the inferior turbinate in the left nasal cavity was seen (Figure 2A). Cervical lymphadenopathy was present on level 1 on the left side. After intranasal incision of the tumour, performed in local anaesthesia, purulent and granular secretion was released. No other abnormalities were seen at physical examination.

Laboratory blood samples showed a C-reactive protein (CRP) of $<1 \mathrm{mg} / \mathrm{L}(\mathrm{n}=<15 \mathrm{mg} / \mathrm{L}$ ) and erythrocyte sedimentation rate (ESR) of $14 \mathrm{~mm} / \mathrm{h}(\mathrm{n}=<15 \mathrm{~mm} / \mathrm{h})$. Pathological assessment of the obtained secretion from the intranasal swelling demonstrated a chronic granulomatous inflammatory process with necrosis. On microscopic inspection, there were no signs of vasculitis, no acid-fast bacilli (AFB) and no bacterial colonies or fungi. The CT- and MRI-scan of the head and neck region showed thickening of the soft tissue in the left nasal cavity, with extension to the lateral side, without destruction of surrounding tissues (Figure 2B). The PET-CT-scan showed increased fludeoxyglucose (FDG) uptake at the site of the nasal lesion and cervical lymph node. A cytological sample of the lymph node demonstrated groups of histiocytic cells, strongly suspected for granuloma with necrosis. A second biopsy of the inner left lateral side of the cavum nasi once again showed a chronic granulomatous inflammation without an identified bacterial, fungal or parasitic pathogen. Treatment with different antibiotics did not relieve symptoms. Four weeks after initial presentation, an infectious disease specialist was consulted by e-mail. He made the working diagnosis of Espundia.

Polymerase Chain Reaction (PCR) test of a new intranasal biopsy led to the diagnosis of Leishmania braziliensis. Treatment was initiated with liposomal amphotericin B (Ambisome), 40 mg/kg intravenously over a period of four weeks. Within these weeks, the nasal tumour decreased and the clinical condition of the patient improved (Figure 3). No adverse effects were observed. At three months follow-up, intranasal endoscopy showed a small adhesion between the inferior turbinate and septum. No remaining tumour was seen (Figure 4A). The CT-scan showed a normal nasal cavity (Figure 4B) apart from the adhesion mentioned above. Adhesiolysis was performed in local anaesthesia and the patient was referred back to his primary care physician.

\section{Discussion}

Nasal obstruction is a common clinical condition in daily ENTpractice. However, obstruction due to mucocutaneous leishmaniasis is seldomly seen in Northern Europe.

Leishmaniasis is a vector-borne infectious disease, caused by many different species of intracellular Leishmania parasites. It is endemic mostly in rural tropical areas in Central- and South America (New World), parts of Africa, India, the Middle East (Old World), but also in parts of Europe, mainly the Mediterranean area ${ }^{(1-4)}$. Over twenty different species of Leishmania can cause disease in humans. It is transmitted by infected female sandflies of the Phlebotominae family. When an infected sandfly bites the human skin, the Leishmania-parasite is released and ingested by macrophages. Within the macrophage, the parasite is hidden from the human immune system and transforms into an amastigote $^{(4,5)}$.

Not every patient who is infected with the Leishmania parasite suffers symptomatic disease: most infections remain subclinical or are self-limiting ${ }^{(3)}$. However, leishmaniasis can lead to a broad spectrum of symptoms that can be classified in three main categories: cutaneous, mucocutaneous (ML) and visceral leishmaniasis. In the case of cutaneous leishmaniasis, the parasitic infection leads to painless ulceration with an indurated border at the location of the bite, usually on exposed areas of the skin. Visceral leishmaniasis, also known as kala-azar, is the most severe form of the disease. The parasite disseminates to the viscera, liver, spleen and bone marrow. Without treatment, visceral leishmaniasis is usually fatal. Mucocutaneous leishmaniasis, also known as Espundia, is most prevalent in South America (Bolivia, Peru, Brazil) ${ }^{(6)}$ and is mainly caused by the Leishmania braziliensis. In 1-10\% of infections with L. braziliensis, clinical symptoms occur ${ }^{(3,7)}$. The parasite disseminates haematologically to the nasopharyngeal mucosa from the location of the sandfly bite ${ }^{(4-6)}$. A Brazilian retrospective study of 327 cases of ML reported involvement of the nasal cavity in $98 \%$ of the cases, followed by pharynx (11\%), oral cavity (6\%) and larynx $(2 \%)^{(7)}$. The most common nasal symptoms are persistent congestion, rhinorrhoea and discharge ${ }^{(3,4,8,9)}$. If left untreated, mucocutaneous leishmaniasis can lead to severe destruction of the skin, soft tissue, bone and potentially sepsis ${ }^{(3,10,11)}$.

Leishmaniasis can be diagnosed by visualisation of LeishmanDonovan bodies, which are the intracellular amastigotes. In the case of nasal mucocutaneous leishmaniasis, these bodies are found in tissue that is obtained through biopsy or aspiration. In our case, these were not detected, not even in retrospect. Alter- 

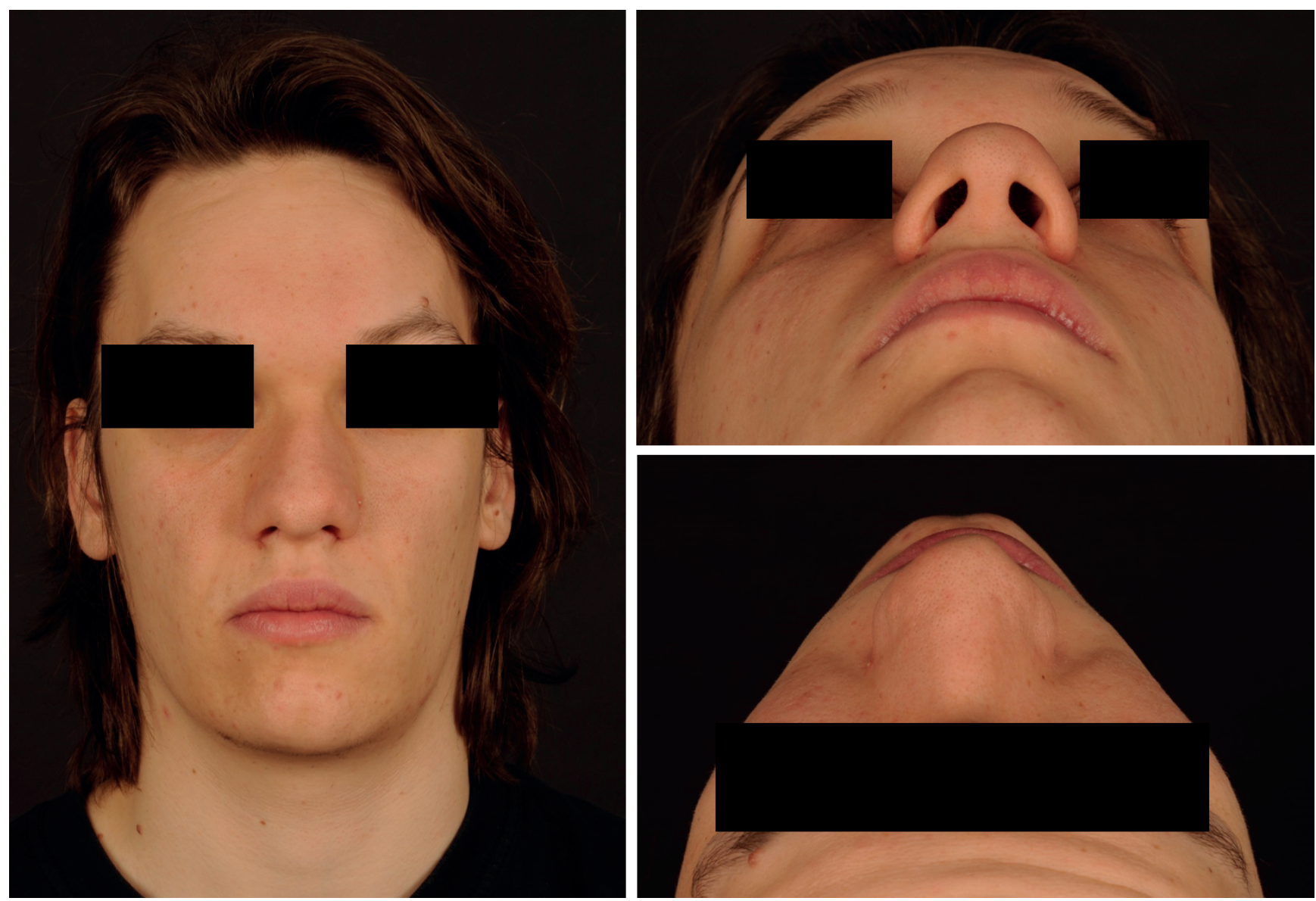

Figure 3. Clinical images taken after treatment with liposomal amphotericin B. Swelling of the left lateral side of the nose has diminished. Minimal asymmetry of the nose is visible.
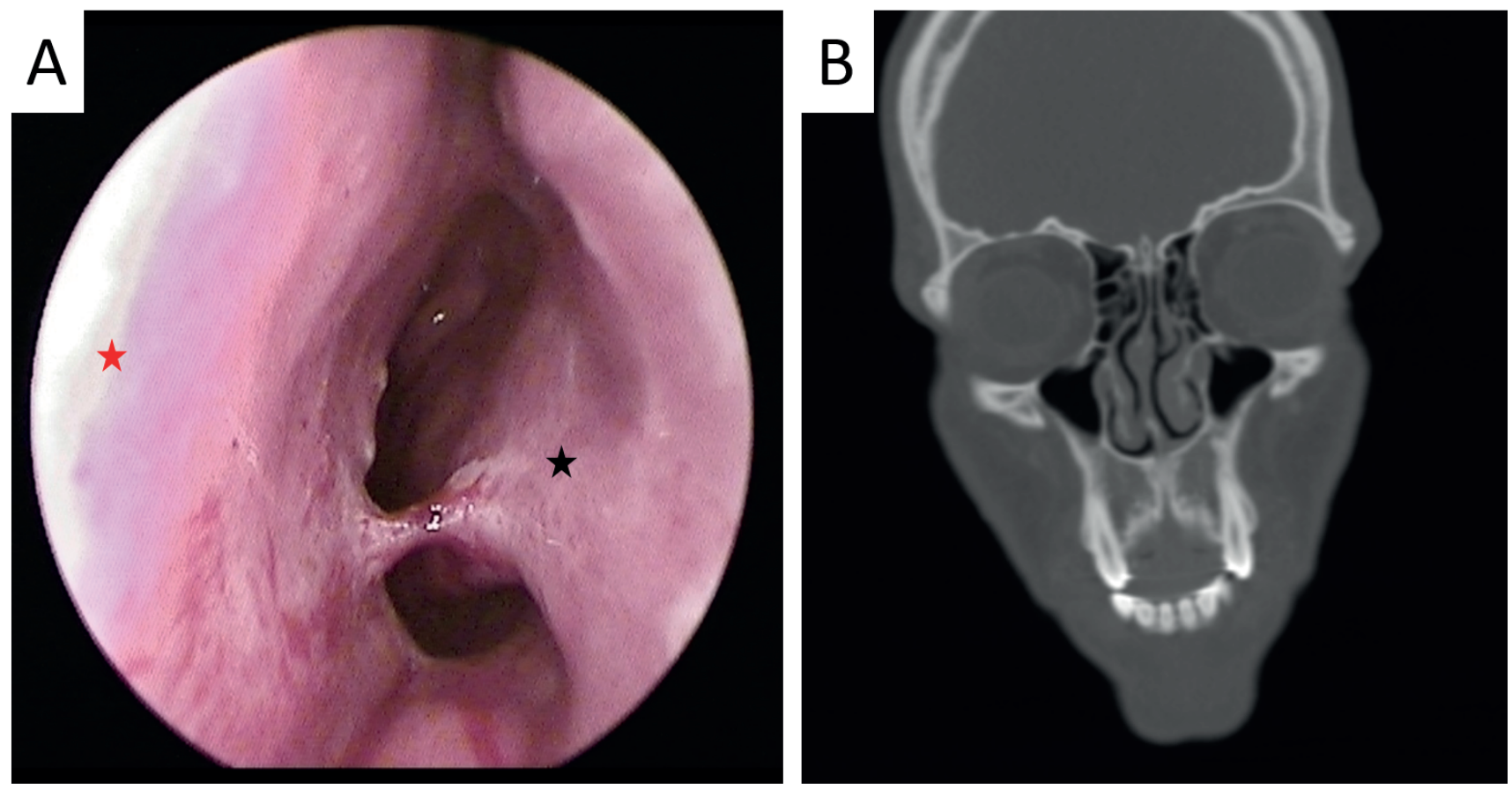

Figure 4. Post-treatment nasal endoscopy and CT-scan at three months follow-up. A. View at nasal endoscopy after treatment. The tumour has disappeared and there is a normal nasal mucosa. A small adhesion is seen. The red asterisk indicates the nasal septum, the black asterisk the inferior turbinate. B. Post-treatment CT-scan of the paranasal sinuses, coronal view. Normal nasal mucosa, no tumour is seen. 
natively, PCR tests can be used to detect Leishmania DNA, which led to the diagnosis in our case.

$M L$ should always be treated because of the risk of a secondary infection or facial deformation ${ }^{(6,7)}$. Due to the localisation and the risk of dissemination of the disease, systemic therapy is required $^{(12)}$. Dutch guidelines advise treatment with antimonybased drugs, a chemical element that interferes with the metabolism of the Leishmania-parasite ${ }^{(13)}$. However, antimony-based drugs can lead to severe nephro- and cardiotoxicity. Alternative treatment options are miltefosine, an oral oncolytic medicine and liposomal Amphotericin B, an antimycotic agent ${ }^{(12)}$. Both of these alternative treatments have proven efficacy against leishmaniasis and although the evidence is poor, seem to be less toxi $^{(14,15)}$. The registration of these drugs differs between European countries. Because miltefosine is currently not registered in the Netherlands, we chose to treat our patient with liposomal Amphotericin B, which is registered, but only for visceral leishmaniasis.

The low prevalence in Europe of leishmaniasis and especially Espundia can lead to a lack of knowledge of this disease among otorhinolaryngologists.

Although the World Health Organization registers the number of reported cases of leishmaniasis in Northern Europe, cases of $M L$ are not registered separately. Therefore, the exact prevalence of $M L$ in Europe is currently unknown. A Swedish study that assessed all microbiologically confirmed leishmaniasis cases between 1996 and 2016, reported four cases of ML in total ${ }^{(11)}$. Recently, case reports of Espundia in Italy, Spain and Belgium have been published ${ }^{(16-18)}$. The lack of knowledge about the disease, may lead to treatment delay. In this case, numerous diagnoses were considered before the final diagnosis was made. Differential diagnoses included tumours of either benign or malignant nature, infectious diseases such as tuberculosis, Lyme borreliosis and rhinosporidiosis, necrosis of lymph nodes and tropical diseases such as filariasis and schistosomiasis. However, it took several weeks before the diagnosis of leishmaniasis was considered and the proper diagnostic tests were applied.

A complicating factor in the diagnostic process is the incubation period of ML: years can pass before a patient becomes symptomatic ${ }^{(10)}$. The relation between a patient's visit to the tropics and the symptoms may not be recognized by the physician or the patient. It is therefore important to thoroughly discuss a patient's travel history, especially in the case of unexplained complaints.

Awareness of this disease is also relevant because of the expected increase incidence of leishmaniasis in Northern Europe. This is partly due to the increasing numbers of patients who travel to endemic areas ${ }^{(1,3,6)}$. Moreover, in the future the sandfly may inhabit parts of Europe other than the Mediterranean region due to climate change $\mathrm{e}^{(3,19,20)}$.

\section{Conclusions}

In conclusion, knowledge of leishmaniasis is of importance for otorhinolaryngologists in Europe, as they will be confronted more frequently with leishmaniasis in the future. Through this case report, we hope to bring the Leishmania-parasite to your attention. Moreover, we emphasize the importance of determining the patient's complete travel history, especially in case of unexplained persisting symptoms.

\section{Acknowledgments}

The authors would like to thank Ms. E.R.F. Baekelandt for proofreading the manuscript.

\section{Authorship contribution}

AS and AW wrote the manuscript and contributed equally. ET,

ES and JK critically revised the manuscript. All authors read and approved the final manuscript.

\section{Conflict of interest}

The authors declare no conflict of interest.

\section{Ethics approval and consent to participate}

Not applicable.

\section{Consent for publication}

Written informed consent for publication of clinical details and clinical images was obtained from the patient. A copy of the consent form is available for review by the Editor of this journal.

\section{Availability of data and materials}

Not applicable.

\section{Funding}

There was no funding of this work.

\section{References}

1. Oryan A, Akbari M. Worldwide risk factors in leishmaniasis. Asian Pac J Trop Med. 2016;9(10):925-32.

2. Davies CR, Kaye P, Croft SL, Sundar S Leishmaniasis: new approaches to disease control. BMJ (Clinical research ed).
2003:326(7385):377-82.

3. Kevric I, Cappel MA, Keeling JH. New World and Old World Leishmania Infections: A Practical Review. Dermatol Clin. 2015;33(3):579-93.

4. Handler MZ, Patel PA, Kapila R, Al-Qubati Y, Schwartz RA. Cutaneous and mucocutane- ous leishmaniasis: Clinical perspectives. J Am Acad Dermatol. 2015;73(6):897-908.

5. Aronson N, Herwaldt BL, Libman M et al. Diagnosis and Treatment of Leishmaniasis: Clinical Practice Guidelines by the Infectious Diseases Society of America (IDSA) and the American Society of Tropical 
Medicine and Hygiene (ASTMH). Am J Trop Med Hyg. 2017;96(1):24-45.

6. Gatti GL, Freda N, Stabile M et al. Deforming Mucocutaneous Leishmaniasis of the Nose. J Craniofac Surg. 2017;28(5):e446-e7.

7. Cincura C, de Lima CMF, Machado PRL et al. Mucosal leishmaniasis: A Retrospective Study of 327 Cases from an Endemic Area of Leishmania (Viannia) braziliensis. Am J Trop Med Hyg. 2017;97(3):761-6.

8. Solomon M, Sahar N, Pavlotzky F et al. Mucosal Leishmaniasis in Travelers with Leishmania braziliensis Complex Returning to Israel. Emerg Infect Dis. 2019;25(4):642-8.

9. Ahluwalia S, Lawn SD, Kanagalingam J, Grant H, Lockwood DN. Mucocutaneous leishmaniasis: an imported infection among travellers to central and South America. Bmj. 2004;329(7470):842-4.

10. Piscopo TV, Mallia Azzopardi C. Leishmaniasis. Postgrad Med J. 2007;83 (976):649-57.

11. Glans H, Dotevall L, Sobirk SK, Farnert A, Bradley M. Cutaneous, mucocutaneous and visceral leishmaniasis in Sweden from 19962016: a retrospective study of clinical characteristics, treatments and outcomes. BMC Infect Dis. 2018;18(1):632.
12. Blum J, Buffet P, Visser L et al. LeishMan recommendations for treatment of cutaneous and mucosal leishmaniasis in travelers, 2014. J Travel Med. 2014;21(2):116-29.

13. Haldar AK, Sen P, Roy S. Use of Antimony in the Treatment of Leishmaniasis: Current Status and Future Directions. Molecular Biology International. 2011;2011:571242.

14. Cunha MA, Leão $A C Q$, de Cassia Soler $R$, Lindoso JAL. Efficacy and Safety of Liposomal Amphotericin B for the Treatment of Mucosal Leishmaniasis from the New World: A Retrospective Study. Am J Trop Med Hyg. 2015;93(6):1214-8.

15. Soto J, Toledo J, Valda $L$ et al. Treatment of Bolivian mucosal leishmaniasis with miltefosine. Clin Infect Dis. 2007:44(3):350-6.

16. Gaspari V, Zaghi I, Macri G et al. Autochthonous Cases of Mucosal Leishmaniasis in Northeastern Italy: Clinical Management and Novel Treatment Approaches. Microorganisms. 2020;8(4).

17. Roca B, Roca M. Mucocutaneous leishmaniasis (espundia). Postgrad Med J. 2020.

18. Menten K, Soentjens P, Caenepeel $P$, Lemkens $P$. Mucocutaneous leishmaniasis of the nose: a case report. B-Ent. 2015:11(1):7780.
19. Aspock H, Gerersdorfer T, Formayer H, Walochnik J. Sandflies and sandfly-borne infections of humans in Central Europe in the light of climate change. Wien Klin Wochenschr. 2008;120(19-20 Suppl 4):24-9.

20. Trájer AJ, Bede-Fazekas Á, Hufnagel L, Horváth L, Bobvos J. The effect of climate change on the potential distribution of the European Phlebotomus species. Applied Ecology and Environmental Research. 2013;11(2):189-208

A.A. Schenck

Department of Otorhinolaryngology

HagaZiekenhuis

The Hague

the Netherlands

Tel: +31 702102696

E-mail:kno@hagaziekenhuis.nl

ISSN: 2589-5613 / ( 2020 The Author(s). This work is licensed under a Creative Commons Attribution 4.0 International License. The images or other third party material in this article are included in the article's Creative Commons license, unless indicated otherwise in the credit line; if the material is not included under the Creative Commons license, users will need to obtain permission from the license holder to reproduce the material. To view a copy of this license, visit http://creativecommons.org/ licenses/by/4.0/ 